\title{
BIOLOGIA E TABELA DE VIDA DE FERTILIDADE DE Spodoptera eridania (CRAMER) (LEPIDOPTERA: NOCTUIDAE) EM MORANGUEIRO E VIDEIRA ${ }^{1}$
}

\author{
LÍGIA CAROLINE BORTOLI ${ }^{2}$, ALINE BERTIN ${ }^{3}$, \\ CAIO FÁBIO STOFFEL EFROM ${ }^{4}$, MARCOS BOTTON ${ }^{5}$
}

RESUMO - A biologia de Spodoptera eridania foi estudada em laboratório $\left(22 \pm 1^{\circ} \mathrm{C}\right.$, UR $70 \pm 10 \%$, fotofase de 14 horas), em folhas de morangueiro (Fragaria x ananassa cv. 'Aromas') e videira (Vitis vinifera cv. 'Cabernet Sauvignon'). A duração e a viabilidade do ciclo total foram, respectivamente, de 52,2 $\pm 1,32$ dias e $37,6 \%$ para morangueiro e $42,2 \pm 0,45$ dias e $25,5 \%$ para videira. A razão sexual em morangueiro foi de 0,58 , e 0,48 em videira. A longevidade média de machos e fêmeas em morangueiro foi de 16,3 $\pm 1,16$ e $15,8 \pm 1,85$ dias, respectivamente, e $5,6 \pm 0,88$ e 7,3 $\pm 0,83$ dias em videira. A fecundidade média total foi de $1.747,5 \pm 187,32$ ovos por fêmea em morangueiro, e 1.764,9 $\pm 289,04$ em videira. A tabela de vida de fertilidade mostrou que a taxa líquida de reprodução e a razão finita de aumento foram de 394,89 e 1,10, respectivamente, para morangueiro, e de 213,98 e 1,12 para videira. As culturas do morangueiro cv. 'Aromas' e da videira cv. 'Cabernet Sauvignon' são hospedeiras favoráveis e equivalentes quanto ao potencial de crescimento populacional de S. eridania.

Termos para indexação: lagarta-das-folhas, Vitis vinifera, Fragaria x ananassa.

\section{BIOLOGY, FERTILITY LIFE TABLE AND EFFECT OF INSECTICIDES ON Spodoptera eridania (CRAMER) (LEPIDOPTERA: NOCTUIDAE) IN STRAWBERRY AND GRAPE}

\begin{abstract}
The biology of Spodoptera eridania was studied in the laboratory $\left(22 \pm 1^{\circ} \mathrm{C}\right.$, RH $70 \pm 10 \%, 14$ hours of photoperiod) in leaves of strawberry (Fragaria $\mathrm{x}$ ananassa cv. 'Aromas') and grape (Vitis vinifera cv. 'Cabernet Sauvignon'). The duration and viability of the entire cycle were respectively $52.2 \pm 1.32$ days and $37.6 \%$ for strawberry and $42.2 \pm 0.45$ days and $25.5 \%$ for grapes. The sex ratio in strawberry was 0.58 and 0.48 on grape. The average longevity of males and females in strawberry was $16.3 \pm 1.16$ and $15.8 \pm 1.85$ days, respectively, and $5.6 \pm 0.88$ and $7.3 \pm 0.83$ days in grape. The mean fecundity was $1,747.5 \pm 187.32$ eggs per female in strawberry and $1,764.9 \pm 289.04$ on grape. The life table of fertility showed that the net reproduction rate and finite rate of increase were 394.89 and 1.10, respectively, for strawberry and 213.98 and 1.12 for grapevine. Strawberry cv. 'Aromas' and grape cv. 'Cabernet Sauvignon' are suitable hosts providing similar population growth rates of S. eridania.
\end{abstract}

Index terms: southern armyworm, Vitis vinifera, Fragaria $\mathrm{x}$ ananassa.

\footnotetext{
'(Trabalho 226-11). Recebido em: 29-08-2011. Aceito para publicação em: 16-10-2012.

${ }^{2}$ Graduanda do curso de Ciências Biológicas da Universidade de Caxias do Sul, Estagiária da Embrapa Uva e Vinho, Lab. de Entomologia, Rua Livramento, 515, CEP 95750-000, Bento Gonçalves-RS. Bolsista CNPq. E-mail: ligia_bortoli@hotmail.com

${ }^{3}$ Biól., Mestranda do curso de Pós-graduação em Entomologia, ESALQ/USP, Laboratório de Biologia de insetos, Av. Pádua Dias, 11, cx. postal 9, CEP 13418-900, Piracicaba, SP,E-mail: aline.bertin.bio@hotmail.com, Bolsista CNPq.

${ }^{4}$ Eng. Agr. Dr. Pós-doutorando Junior, Embrapa Uva e Vinho, Lab. de Entomologia, Rua Livramento, 515, CEP 95750-000, Bento Gonçalves-RS. Bolsista CNPq. E-mail: caioefrom@hotmail.com

${ }^{5}$ Eng. Agr. Dr. Pesquisador, Embrapa Uva e Vinho, Lab. de Entomologia, Rua Livramento, 515, CEP 95750-000, Bento Gonçalves, RS, E-mail: marcos@cnpuv.embrapa.br
} 


\section{INTRODUÇÃO}

A lagarta-das-folhas, Spodoptera eridania (Cramer) (Lepidoptera: Noctuidae), era considerada uma praga secundária em diversos cultivos, incluindo espécies anuais e perenes (FONSECA, 2006). Entretanto, nos últimos anos, a espécie tem sido relatada como praga importante nas culturas do algodão e soja (SANTOS et al., 2005; SANTOS et al., 2010), tomate (MIRANDA et al., 2005) e frutífe

ras de clima temperado, com destaque para a macieira (NORA et al., 1989; FONSECA, 2006). No Estado do Rio Grande do Sul, S. eridania tem sido encontrada causando prejuízos importantes também em morangueiro e videira, resultando na necessidade de aplicação de inseticidas durante a safra.

Os danos em morangueiro e videira decorrem da alimentação das lagartas em folhas e nos frutos, depreciando-os comercialmente. $\mathrm{O}$ dano causado pela praga pode também servir de porta de entrada para patógenos, como podridões, além de provocar a fermentação dos frutos, atraindo pragas secundárias, como os nitidulídeos.

S. eridania é uma espécie polífaga, sendo que sua biologia já foi estudada em folhas de algodoeiro (Gossypium spp. L.), soja (Glycine max L.) e corda-de-viola (Ipomoea grandifolia (Dammer) O'Donell), sendo esta última considerada um hospedeiro alternativo (SANTOS et al., 2005). Já, Mattana e Foerster (1988a,b) constataram que lagartas de $S$. eridania são capazes de se desenvolver e reproduzir em bracatinga (Mimosa scabrella Benth) e batata-doce (Ipomoea batatas L.) e, apesar de as lagartas possuírem desenvolvimento mais lento, menor peso pupal e menor razão de crescimento, quando comparadas às criadas em folhas de batata-doce, podem causar prejuízos significativos em reflorestamentos. No entanto, em frutíferas de clima temperado e na videira, não existem informações sobre a biologia da espécie, prejudicando a implantação de estratégias de manejo integrado de pragas. Neste trabalho, foi estudada a biologia e elaborada a tabela de vida de fertilidade de $S$. eridania em morangueiro e videira.

\section{MATERIAL E MÉTODOS}

O trabalho foi realizado no Laboratório de Entomologia da Embrapa Uva e Vinho, em Bento Gonçalves-RS, em sala climatizada com temperatura de $22 \pm 1^{\circ} \mathrm{C}, 70 \pm 10 \%$ de umidade relativa do ar e fotofase de 14 horas. Para os bioensaios, lagartas de S. eridania foram coletadas de plantas invasoras, em um vinhedo localizado no município de Bento Gonçalves-RS $\left(29^{\circ} 06^{\prime} 55^{\prime \prime} \mathrm{S}\right.$ e $51^{\circ} 26^{\prime} 50^{\prime \prime}$ $\mathrm{O} ; 640 \mathrm{~m}$ de altitude). As lagartas foram trazidas ao laboratório onde foram criadas até a fase adulta, sendo identificadas pelo Dr. Alexandre Specht, da Universidade de Caxias do Sul. Juntamente com as lagartas coletadas, foram identificados parasitoides emergidos, que foram identificados pela Dra. Angélica Martins Dias, da Universidade Federal de São Carlos.

Em laboratório, as lagartas provenientes dos ovos dos indivíduos coletados no campo foram utilizadas para estudar a biologia em morangueiro (Fragaria x ananassa Duch.) cultivar 'Aromas' e videira (Vitis vinifera L.) cultivar 'Cabernet Sauvignon'. As folhas de videira utilizadas nos experimentos foram obtidas de plantas de 10 anos, da área experimental da Embrapa Uva e Vinho, Bento Gonçalves-RS, e as de morangueiro em plantas de um ano, mantidas em casa de vegetação da mesma instituição.

Os parâmetros biológicos avaliados para as duas culturas foram: período de incubação e viabilidade dos ovos, duração e viabilidade das fases larval e pupal, número de instares, peso de pupas machos e fêmeas com 24 horas de idade, razão sexual, longevidade dos adultos, período de préoviposição, oviposição, pós-oviposição, fecundidade das fêmeas e a tabela de vida de fertilidade.

Para a obtenção dos parâmetros da fase larval, foram individualizadas 120 lagartas recémeclodidas em potes plásticos $(10 \mathrm{~cm}$ de diâmetro $\mathrm{X}$ $6 \mathrm{~cm}$ de altura) contendo papel- filtro umedecido e folhas de morangueiro e videira. Destas 120 lagartas, foram selecionadas 25 lagartas para medição da cápsula cefálica, sendo os dados utilizados para a formulação das hipóteses e determinação do número de instares. As medições foram feitas diariamente por meio de ocular graduada acoplada ao microscópio estereoscópico. A duração e a viabilidade da fase larval foram calculadas com base nos dados obtidos dos 120 indivíduos.

As pupas obtidas foram transferidas para tubos de vidro, de fundo chato $(2,5 \mathrm{~cm}$ de diâmetro $\mathrm{X} 8 \mathrm{~cm}$ de altura), contendo papel-filtro umedecido, onde permaneceram até a emergência dos adultos. As pupas foram sexadas segundo Butt e Cantu (1962), pesadas após 24 horas, e foi determinada a proporção sexual.

Dos adultos obtidos, foram formados casais com mesma idade $(n=17)$, individualizados em gaiolas de garrafa pet e alimentados com solução aquosa de mel a $15 \%$, para a obtenção dos dados sobre longevidade, período de pré-oviposição, oviposição, pós-oviposição e fecundidade das 
fêmeas.

Ovos da segunda postura de 12 fêmeas foram separados e mantidos em placas de Petri sobre papel-filtro umedecido. Foram registrados o número de lagartas eclodidas e a duração do período embrionário destes indivíduos.

Os dados obtidos em cada fase, dos ensaios de biologia, foram submetidos à análise de variância; e as médias, comparadas pelo teste de Tukey, a 5\% de probabilidade, pelo programa SPSS 15. Apartir dos dados biológicos, elaborou-se a tabela de vida de fertilidade, estimando-se a duração média de uma geração (T), a taxa líquida de reprodução (Ro), a taxa intrínseca de aumento (Rm) e a razão finita de aumento $(\lambda)$, sendo as médias comparadas pelo teste " $\mathrm{t}$ " unilateral, a $5 \%$ de probabilidade, utilizando o programa "SAS System", conforme Maia et al. (2000).

\section{RESULTADOS E DISCUSSÃO}

De uma amostra de 100 pupas provenientes das lagartas coletadas a campo para o estabelecimento dos ensaios em laboratório, emergiram 15 parasitoides dos gêneros Colpotrochia sp. (Hymenoptera, Ichneumonidae) $(\mathrm{n}=13)$ e Ophion sp. $(\mathrm{n}=2)$ (Hymenoptera, Ichneumonidae). Os dois gêneros já foram relatados parasitando Spodoptera eridania (Cramer) e Spodoptera frugiperda (J. E. Smith) (Lepidoptera, Noctuidade) em outras regiões, indicando serem parasitoides também presentes em pomares da região (TINGLE et al., 1978; DEQUECH et al., 2004).

O período de incubação e a viabilidade dos ovos de $S$. eridania diferiram $(\mathrm{P}<0,01)$ quando as lagartas foram criadas em morangueiro $(5,1$ dias e $71,7 \%)$ e videira $(4,4$ dias $52,38 \%$ ) (Tabela 1$)$. Foerster e Dionísio (1989) obtiveram período de incubação de seis dias, à temperatura de $20^{\circ} \mathrm{C}$ em bracatinga. Santos et al. (2005), à temperatura de $27^{\circ} \mathrm{C}$, obtiveram valores variando de 3,2 a 3,3 dias para algodoeiro, corda-de-viola e soja. A viabilidade dos ovos em morangueiro foi superior quando comparada à observada por Parra et al. (1977) em soja e algodão, que foi de $58,57 \%$ e $47,89 \%$, respectivamente, embora estas sejam semelhantes às obtidas para videira. Considerando as duas espécies vegetais, as viabilidades foram inferiores às encontradas por Santos et al. (2005), com valores de 81,$4 ; 74,4$ e $81,1 \%$ para algodoeiro, corda-deviola e soja.

A duração do estágio larval foi de 34,6 dias em morangueiro (Tabela 1), resultado próximo ao encontrado por Mattana e Foerster (1988a) de
32,04 dias a $25^{\circ} \mathrm{C}$ em bracatinga. Entretanto, a duração em videira diferiu estatisticamente, com 26,7 dias $(\mathrm{P}<0,0001)$, explicitando as alterações ocasionadas no ciclo biológico devido às diferenças entre hospedeiros. A viabilidade obtida foi de $60 \%$ para morangueiro e $66,25 \%$ para videira, inferiores ao relatado por Foerster e Dionísio (1989) em bracatinga $(86,2 \%)$, à temperatura de $20^{\circ} \mathrm{C}$, por Parra et al. (1977) em soja e algodoeiro, onde a viabilidade em ambos hospedeiros foi de $96 \%$ e por Santos et al. (2005), com viabilidade superior a $80 \%$ em soja, algodoeiro e corda-de-viola. A baixa viabilidade observada em morangueiro e videira sugere que estes hospedeiros não proporcionaram melhor desenvolvimento à espécie quando comparado aos vegetais já estudados, possivelmente devido a uma inadequação nutricional. No desenvolvimento larval de S. eridania, foram observados seis instares, concordando com os resultados obtidos por Parra et al. (1977) e Santos et al. (2005) em algodoeiro e Mattana e Foerster (1988b) em batata-doce.

A duração média da fase de pupa foi de 12,2 (morangueiro) e 11,9 dias (videira) $(\mathrm{P}=0,3107)$ (Tabela 1), com diferença de um dia entre machos e fêmeas nas duas espécies (morangueiro: machos - 12,6 e fêmeas - 11,5 dias; videira: machos - 12,4 e fêmeas - 11,3 dias). O prolongamento do período pupal de machos também foi observado por Santos et al. (2005) e Parra et al. (1977), ambos em algodoeiro e soja. As viabilidades obtidas foram de $87,5 \%$ (morangueiro) e 73,6\% (videira), sendo inferiores ao valor encontrado por Santos et al. (2005), de $93,3 \%$ em algodoeiro, porém superiores à soja (68,3\%). Quanto ao peso médio de pupas com 24 horas de idade, observou-se que as fêmeas foram significativamente mais pesadas que os machos, nos dois hospedeiros (Tabela 2), sendo a média dos dois sexos de $255,0 \mathrm{mg}$ (morangueiro) e $305,7 \mathrm{mg}$ (videira). Parra et al. (1977) e Santos et al. (2005) obtiveram resultados inferiores quanto ao peso de pupas para ambos os sexos em soja. No entanto, os dois autores encontraram valores semelhantes aos verificados no morangueiro em algodão, porém ainda inferiores ao peso de pupas em videira. A razão sexual foi diferente entre as duas espécies, registrando-se maior proporção de fêmeas $(0,58)$ em morangueiro e menor $(0,48)$ em videira. Santos et al. (2005) encontraram valores semelhantes aos obtidos para videira, de 0,41 em algodoeiro, 0,47 em corda-de-viola e 0,46 em soja.

A longevidade média de machos e fêmeas não diferiu significativamente entre si nas duas cultivares (Tabela 3), divergindo dos trabalhos de Santos et al. (2005) e Parra et al. (1977), nos 
quais os machos apresentaram menor longevidade. Entretanto, quando os valores médios dos gêneros foram comparados entre as variedades, foi observada menor longevidade para os adultos oriundos de videira $(\mathrm{P}<0,0001)$. Da mesma forma, quando comparadas as longevidades obtidas por Santos et al. (2005) em soja, algodoeiro e corda-de-viola, os adultos alimentados com folhas de morango foram superiores a estes hospedeiros, o que pode indicar melhor valor nutricional do morangueiro.

Os períodos de pré-oviposição de $S$. eridania foram de 2,6 dias em morangueiro e 2,0 dias para videira $(\mathrm{P}=0,058)$, valores superiores ao observado por Mattana e Foerster (1988a) para bracatinga e batata-doce, que foram de 1,2 e 1,9 dia, respectivamente. $\mathrm{O}$ período de oviposição em morangueiro foi de 8,7 dias (Tabela 3), resultado próximo dos 8,4 dias encontrados por Mattana e Foerster (1988a) em batata-doce. Porém, para videira, ocorreu diferença no período de oviposição, que foi de 5,1 dias $(\mathrm{P}=0,006)$, mais próximo dos valores verificados por Santos et al. (2005) em algodoeiro e soja, 4,4 e 4,2 dias, respectivamente. A duração do período de pós-oviposição em morangueiro, de 5,3 dias, foi superior aos valores constatados por Mattana e Foerster (1988a) para batata-doce (2,6 dias), bracatinga (3,36 dias) e o 1,3 dia em videira, apesar de não ser constatada diferença estatística $(\mathrm{P}=0,8792)$, provavelmente devido à grande variação nos valores obtidos em morangueiro. A duração e a viabilidade do ciclo total (ovo-adulto) diferiram entre os hospedeiros e foram, respectivamente, de 52,2 dias e 37,6\% para morangueiro e, para videira, 42,2 dias e $25,5 \%$
(Tabela 1) $(\mathrm{P}<0,001)$, valores abaixo do verificado por Foerster e Dionísio (1989) para a duração do ciclo total em bracatinga, 60 dias a $20^{\circ} \mathrm{C}$. A viabilidade do ciclo total foi superior à obtida por Santos et al. (2005) em soja, $16,6 \%$, mas inferior à viabilidade em algodoeiro, que foi de $57,7 \%$, sugerindo maior adaptação da praga ao algodoeiro do que ao morangueiro e à videira. Em relação à fecundidade média das fêmeas, foi observada a média de $1.747,5$ ovos por fêmea criada em morangueiro e de $1.764,9$ ovos por fêmea em videira (Tabela 3 ), valores considerados altos se comparados aos 680,5 ovos/fêmea obtidos por Santos et al. (2005) em algodoeiro. A fecundidade média ficou próxima também dos 1.346,0 ovos/fêmea em soja (PARRA et al., 1977) e 1.859,6 ovos/fêmea em batata-doce (MATTANA; FOERSTER, 1988a).

A taxa líquida de reprodução (Ro) indicou que a população de $S$. eridania, quando teve como hospedeiro o morangueiro, pode aumentar 394,89 vezes a cada geração. Já em videira, esse valor é de 213,98 vezes (Tabela 4). O tempo médio de uma geração (T) foi de aproximadamente 59,1 (morangueiro) e 48,8 dias (videira) (Tabela 4). A taxa intrínseca de crescimento $\left(\mathrm{r}_{\mathrm{m}}\right)$ foi de 0,101 (morangueiro) e de 0,110 (videira), e a razão finita de aumento $(\lambda)$, que corresponde ao número de indivíduos adicionados à população, por fêmea por dia, foi de 1,10 em morangueiro e 1,12 em videira. Os resultados obtidos demonstram, claramente, que as culturas do morangueiro cv. 'Aromas' e da videira da cv. 'Cabernet Sauvignon' são hospedeiras adequadas ao completo desenvolvimento de S. eridania, não se diferenciando significativamente quanto à razão finita de aumento da população (Tabela 4).

TABELA 1 - Duração (média \pm EP) e viabilidade (\%) das fases de ovo, lagarta, pupa e ciclo total (ovoadulto) de Spodoptera eridania mantidas em folhas de morangueiro cv. 'Aromas' e videira cv. 'Cabernet Sauvignon'. Temperatura de $22 \pm 1^{\circ} \mathrm{C}$, UR $70 \pm 10 \%$ e fotofase de 14 horas.

\begin{tabular}{lcccc}
\hline \multirow{2}{*}{ Fase } & \multicolumn{2}{c}{ Morangueiro } & \multicolumn{2}{c}{ Videira } \\
\cline { 2 - 5 } & Duração (dias)* & Viabilidade (\%) & Duração (dias) & Viabilidade (\%) \\
\hline Ovo & $5,1 \pm 0,03 \mathrm{a}$ & 71,70 & $4,4 \pm 0,16 \mathrm{~b}$ & 52,38 \\
Lagarta & $34,6 \pm 0,55 \mathrm{a}$ & 60,00 & $26,7 \pm 0,42 \mathrm{~b}$ & 66,25 \\
Pupa & $12,2 \pm 0,16 \mathrm{a}$ & 87,50 & $11,9 \pm 0,44 \mathrm{a}$ & 73,58 \\
Ciclo (ovo-adulto) & $52,2 \pm 1,32 \mathrm{a}$ & 37,60 & $42,2 \pm 0,45 \mathrm{~b}$ & 25,53 \\
\hline
\end{tabular}

*Médias seguidas de letras diferentes, nas linhas, diferem entre si (Tukey 5\%). 
TABELA 2 - Peso médio de pupas (média \pm EP) de Spodoptera eridania mantidas em folhas de morangueiro cv. 'Aromas' e videira cv. 'Cabernet Sauvignon' ( $\mathrm{n}=$ número de observações). Temperatura de $22 \pm 1^{\circ} \mathrm{C}$, UR $70 \pm 10 \%$ e fotofase de 14 horas.

\begin{tabular}{|c|c|c|c|c|}
\hline \multirow{3}{*}{ Sexo } & \multicolumn{2}{|c|}{ Morangueiro } & \multicolumn{2}{|c|}{ Videira } \\
\hline & \multicolumn{4}{|c|}{ Peso $(\mathrm{mg})$} \\
\hline & Média (n)* & Intervalo & Média (n) & Intervalo \\
\hline Fêmea & $266,8 \pm 6,28 \mathrm{Ab}(42)$ & $136-340$ & $343,2 \pm 8,16$ Аa (19) & $282-408$ \\
\hline Macho & $239,0 \pm 4,60 \mathrm{Bb}(31)$ & $167-280$ & $270,1 \pm 6,20 \mathrm{Ba}(20)$ & $215-311$ \\
\hline Média machos e fêmeas & $255,0 \pm 6,44 b(73)$ & $136-340$ & $305,7 \pm 8,16 \mathrm{a}(39)$ & $215-408$ \\
\hline
\end{tabular}

TABELA 3 - Médias de longevidade de machos e fêmeas, período de pré-oviposição, período de oviposição, período de pós-oviposição (dias) ( \pm EP) e número de ovos por fêmea (média $\pm \mathrm{EP}$ ) de Spodoptera eridania mantidas em folhas de morangueiro cv. 'Aromas' e videira cv. 'Cabernet Sauvignon'. Temperatura de $22 \pm 1{ }^{\circ} \mathrm{C}$, UR $70 \pm 10 \%$ e fotofase de 14 horas.

\begin{tabular}{lcc}
\hline \multicolumn{1}{c}{ Fase de adulto* } & Morangueiro & Videira \\
\hline Longevidade de machos & $16,3 \pm 1,16 \mathrm{a}$ & $5,6 \pm 0,88 \mathrm{~b}$ \\
Longevidade de fêmeas & $15,8 \pm 1,85 \mathrm{a}$ & $7,3 \pm 0,83 \mathrm{~b}$ \\
Período de pré-oviposição & $2,6 \pm 0,28 \mathrm{a}$ & $2,0 \pm 0,15 \mathrm{a}$ \\
Período de oviposição & $8,7 \pm 0,75 \mathrm{a}$ & $5,1 \pm 0,73 \mathrm{~b}$ \\
Período de pós-oviposição & $5,3 \pm 2,45 \mathrm{a}$ & $1,3 \pm 0,12 \mathrm{a}$ \\
Fecundidade & $1747,5 \pm 187,32 \mathrm{a}$ & $1764,9 \pm 289,04 \mathrm{a}$ \\
\hline
\end{tabular}

*Médias seguidas de letras diferentes, nas linhas, diferem entre si (Tukey 5\%).

TABELA 4 - Duração média de uma geração $(\mathrm{T})$, taxa líquida de reprodução $\left(\mathrm{R}_{0}\right)$, taxa intrínseca de aumento $(\mathrm{Rm})$ e razão finita de aumento $(\lambda)( \pm \mathrm{EP})$ para indivíduos de Spodoptera eridania mantidos em folhas de morangueiro cv. 'Aromas' e videira cv. 'Cabernet Sauvignon'. Temperatura de $22 \pm 1{ }^{\circ} \mathrm{C}$, UR $70 \pm 10 \%$ e fotofase de 14 horas.

\begin{tabular}{ccccc}
\hline & T (dias) & Ro & Rm & $\begin{array}{c}\lambda \\
\text { (indivíduos/o/dia) }\end{array}$ \\
\hline Morangueiro & $59,1 \pm 0,685 \mathrm{a}$ & $394,89 \pm 27,632 \mathrm{a}$ & $0,101 \pm 0,005 \mathrm{a}$ & $1,10 \pm 0,008 \mathrm{a}$ \\
Videira & $48,8 \pm 0,442 \mathrm{~b}$ & $213,98 \pm 19,785 \mathrm{~b}$ & $0,110 \pm 0,006 \mathrm{a}$ & $1,12 \pm 0,006 \mathrm{a}$ \\
\hline
\end{tabular}

*Médias seguidas de letras diferentes, nas colunas, diferem entre si pelo teste " $\mathrm{t}$ " unilateral a 5\%.

\section{CONCLUSÃO}

As culturas do morangueiro cv. 'Aromas' e da videira cv. 'Cabernet Sauvignon' são hospedeiras favoráveis e equivalentes quanto ao potencial de crescimento populacional de S. eridania.

\section{AGRADECIMENTOS}

Ao CNPq, pelas bolsas concedidas aos autores. À Msc. Cristiane Müller, pelo auxílio na metodologia para os estudos de biologia da espécie no laboratório.

\section{REFERÊNCIAS}

BUTT, B.A.; CANTU, E. Sex determination of lepidopterous pupae. Washington: USDA, 1962. $7 \mathrm{p}$.

DEQUECH, S. T. B.; SILVA, R. P.; FIUZA, L.M. Ocorrência de parasitóides de Spodoptera frugiperda (J. E. Smith)(Lep., Noctuidae) em lavouras de milho em Cachoeirinha, RS. Ciência Rural, Santa Maria, v. 34, p. 1235-1237, 2004. 
FOERSTER, L.A.; DIONÍSIO, A.L.M. Necessidades térmicas de Spodoptera eridania (Cramer, 1782) (Lepidoptera: Noctuidae) em Bracatinga (Mimosa scabrella Bentham) (Leguminosae). Anais da Sociedade Entomológica do Brasil, Jaboticabal, v. 18, p. 145-154, 1989.

FONSECA, F. L. da. Ocorrência, monitoramento, caracterização de danos e parasitismo de noctuidae e geometridae em pomares comerciais de macieira em Vacaria, Rio Grande do Sul, Brasil. 2006. 97f. Tese (Doutorado em Entomologia) -Universidade Federal do Paraná, Curitiba, 2006.

MAIA, H.N.M.; LUIZ, A.J.B.; CAMPANHOLA, C. Statistical inference on associated fertility life table parameters using jackknife technique: computational aspects. Journal of Economic Entomology, Lanham, v.93, n.2, p. 511-518, 2000.

MATTANA, A.L.; FOERSTER, L.A. Ciclo de vida de Spodoptera eridania (Cramer, 1782) (Lepidoptera: Noctuidae) em um novo hospedeiro, Bracatinga (Mimosa scabrella Bentham) (Leguminosae). Anais da Sociedade Entomológica do Brasil, Jaboticabal, v. 17, p. 173-183, 1988a.

MATTANA, A.L.; FOERSTER, L.A. Consumo e utilização de bracatinga (Mimosa scabrella, Bentham) (Leguminosae) e batata doce (Ipomoea batatas L.) (Convolvulaceae) por larvas de Spodoptera eridania (Cramer, 1782) (Lepidoptera: Noctuidae). Anais da Sociedade Entomológica do Brasil, Jaboticabal, v. 17, p. $95-105,1988$ b.
MIRANDA, M. M. M.; PICANÇO, M. C.; ZANUNCIO, J.C.; BACCI, L.; SILVA, E. M. da. Impact of integrated pest management on the population of leafminers, fruit borers, and natural enemies in tomato. Ciência Rural, Santa Maria, v. 35, p. 204-208, 2005.

NORA, I.; REIS FILHO, W.; STUKER, H. Danos de lagartas em frutos e folhas da macieira: mudanças no agroecosistema ocasionam o surgimento de insetos indesejados nos pomares. Agropecuária Catarinense, Florianópolis, v. 2, p. 54-55, 1989.

PARRA, J.R.P.; PRECETTI, A.A.C.M.; KARSTEN JR., P. Aspectos biológicos de Spodoptera eridania (Cramer, 1782) (Lepidoptera: Noctuidae) em soja e algodão. Anais da Sociedade Entomológica do Brasil, Jaboticabal, v. 6, p. 147-155, 1977.

SANTOS, K.B.D.; MENEGUM, A. M.; NEVES, P.M.O.J. Biologia de Spodoptera eridania (Cramer) (Lepidoptera: Noctuidae) em diferentes hospedeiros. Neotropical Entomology, Londrina, v. 34, n. 6, p. 903-910, 2005.

SANTOS, K.B.D.; MENEGUM, A. M.; SANTOS, W. J. dos; NEVES, P.M.O.J.; SANTOS, R.B. dos. Caracterização dos danos de Spodoptera eridania (Cramer) e Spodoptera cosmioides (Walker) (Lepidoptera: Noctuidae) a estruturas de algodoeiro. Neotropical Entomology, Londrina, v. 39, p. 626-631, 2010.

TINGLE, F. C.; ASHLEY, T. R.; MITCHELL, E. R. Parasites of Spodoptera exigua, S. eridania [Lep.: Noctuidae] and Herpetogramma bipunctalis [Lep.: Pyralidae] collected from Amaranthus hybridus in field corn. Biocontrol, Dordrecht, v. 23, p. 343-347, 1978. 